\title{
The effect of personality measurement conditions on spontaneous swimming behavior in the pale chub Zacco platypus (Cyprinidae)
}

\author{
Shi-Jian Fu ${ }^{\text {Corresp. } 1}$ \\ ${ }^{1}$ Laboratory of Evolutionary Physiology and Behavior, Chongqing Key Laboratory of Animal Biology, Chongqing Normal University, Chongqing, China \\ Corresponding Author: Shi-Jian Fu \\ Email address: shijianfu9@cqnu.edu.cn
}

Studies on personality have revealed that some personality traits are strongly correlated; thus, researchers may be able to acquire data for variables related to different personality traits from one measurement. Therefore, the aim of the present study was to test whether spontaneous movement traits used in fish personality measurements are correlated or vary among different contexts in a common Chinese cyprinid fish, the pale chub (Zacco platypus, Cyprinidae). The median swimming speed, percent time spent moving and median turning rate were measured in a boldness context (with a shelter available), then in an exploration context (with a novel object nearby) and finally in a control context (i.e., with no shelter or novel object). The median swimming speed, percent time spent moving, and median turning rate all showed positive correlations between the control and the other two contexts, which suggests that future studies might use spontaneous swimming variables measured in exploration or boldness contexts to avoid the need to carry out a separate activity test. Further analysis comparing the distance to and latency to explore the novel object between the exploration context (with the novel object present) and control context (with an imaginary object at the same position) showed that the amount of time it took for the fish to first reach the object for exploration was significantly shorter in an exploration context than in a control context. This suggests that latency to explore might be useful as a variable indicating exploration in the pale chub in the future. 
2 The effect of personality measurement conditions on

3 spontaneous swimming behavior in the pale chub Zacco

4 platypus (Cyprinidae)

5

6

9 Laboratory of Evolutionary Physiology and Behavior, Chongqing Key

10 Laboratory of Animal Biology, Chongqing Normal University, Chongqing,

11 401331, China

12

$14{ }^{*}$ Corresponding author

15 E-mail: shijianfu9@cqnu.edu.cn; Tel. and Fax: 862365910701

17 Running title: Spontaneous activity and personality 


\section{ABSTRACT}

Studies on personality have revealed that some personality traits are strongly correlated; thus, researchers may be able to acquire data for variables related to different personality traits from one measurement. Therefore, the aim of the present study was to test whether spontaneous movement traits used in fish personality measurements are correlated or vary among different contexts in a common Chinese cyprinid fish, the pale chub (Zacco platypus, Cyprinidae). The median swimming speed, percent time spent moving and median turning rate were measured in a boldness context (with a shelter available), then in an exploration context (with a novel object nearby) and finally in a control context (i.e., with no shelter or novel object). The median swimming speed, percent time spent moving, and median turning rate all showed positive correlations between the control and the other two contexts, which suggests that future studies might use spontaneous swimming variables measured in exploration or boldness contexts to avoid the need to carry out a separate activity test. Further analysis comparing the distance to and latency to explore the novel object between the exploration context (with the novel object present) and control context (with an imaginary object at the same position) showed that the amount of time it took for the fish to first reach the object for exploration was significantly shorter in an exploration context than in a control context. This suggests that latency to explore might be useful as a variable indicating exploration in the pale chub in the future.

Keywords personality measurement context, temperament, behavioral syndrome, activity, boldness, exploration

\section{INTRODUCTION}

Animal personality is measured as a consistent difference among individuals in behaviors such as boldness, exploration and activity (Sih, Bell \& Johnson, 2004; Bell, Hankison \& Laskowski, 2009; Réale et al., 2010; Mazué \& Godin, 2015; Jolles et al., 2019). Personality has been assumed to have large fitness consequences and a wide range of ecological and evolutionary implications (Smith \& Blumstein, 2008; Réale et al., 2010; Sih et al., 2012); hence, it has attracted much attention recently (Jolles et al., 2017; Tang \& Fu, 2019). The tendency to leave a refuge and search for an open environment is traditionally referred to as boldness (Burns, 2008; Jolles et al., 2017), and studies have used latency to leave the shelter and percentage of time (or activity) outside the shelter as indicators 
46

of this characteristic (Brown \& Irving, 2014; Bevan et al., 2018; Tang, Wu \& Fu, 2018). The novel object test has been widely used in personality research to assess exploratory behavior (i.e., curiosity towards novelty) and neophobia (i.e., fear of novelty) (Galhardo, Vitorino \& Oliveira, 2012). Exploration is usually evaluated in terms of the distance or latency associated with the inspection of a novel object (Liu \& Fu, 2017). For activity measurements, fish biologists usually use the characteristics of spontaneous activities (movements without external stimulus) in an arena, for example, the median swimming speed and percent time moving during a given period (Brown \& Irving, 2014; Liu \& Fu, 2017). It has long been recognized that many animals exhibit a so-called 'behavioral syndromes', as bold individuals are typically more exploratory and active than other individuals (Sih, Bell \& Johnson, 2004; Sih et al., 2012; Martins \& Bhat, 2014); i.e., personality traits are highly correlated, at least with regard to boldness, exploration and activity. A study involving principal component analysis also suggested that most variables can be reduced to a so-called 'activity component', which is distinct from a 'sociability component' (Tang, 2019). If the variables are correlated across different personality contexts, researchers may be able to acquire data for variables related to different personality traits from one measurement. For example, one could measure the activity variables mentioned above from spontaneous movement trajectories of fish being tested in a boldness or an exploration context. Thus, the present study aimed to determine whether swimming behavior traits were correlated within individuals across different personality contexts. The contexts included a boldness context with a shelter available, an exploration context with a novel object nearby and an activity context with no novel object or shelter. I tested the correlations between the activity (i.e., control) context and the other two contexts but did not test the correlation between the boldness and exploration contexts because the test conditions were not strictly controlled between these two contexts; i.e., in the boldness context, there was a shelter nearby but no novel object, whereas in the exploration context, there was a novel object but no shelter nearby.

To achieve these goals, I selected the pale chub (Zacco platypus), a common small cyprinid fish species distributed across East Asia, as an experimental model. This omnivorous species often forms shoals in open water but may also be found solitarily, refuging in plants or behind stones when foraging 
or avoiding predators. I recorded videos of individual fish in different personality contexts with a webcam. The variables I selected for comparison were median swimming speed, percent time spent moving, and median turning rate of the body centroid (which usually serves as an indicator of exploration tendency but is also strongly correlated with activity) (Couzin et al., 2011; loannou, Singh \& Couzin, 2015; Sumpter et al., 2018).

To further test whether the novel object used in the present study was a sufficient stimulus for exploration in the pale chub, I conducted an additional analysis comparing the mean distance to the novel object and the latency to explore the novel object in the exploration context with similar measures in relation to a virtual object in the activity context. The aim of this additional analysis was to test whether the novel object was an adequate stimulus or whether the correlation between variables of activity and exploration was an artifact of the measurement protocols. For example, more active fish (individuals that spend more time moving and with a faster speed) might by chance take less time to reach a novel object independent of their exploration tendency. Because no investigation has been conducted to test this issue, it might provide a useful result for studies of exploration measurements in the future.

\section{MATERIAL AND METHODS}

\section{Source of the fish and their care}

A total of 80 juvenile pale chubs (body mass: $6.42 \pm 1.26 \mathrm{~g}$; mean \pm S.D.) were captured by local fishers from a tributary of the Wujiang River $\left(29^{\circ} 24^{\prime} 37^{\prime \prime} \mathrm{N}, 107^{\circ} 31^{\prime} 55^{\prime \prime} \mathrm{E}\right.$, Wulong County, Chongqing city). All fish were held in a $250 \mathrm{~L}$ recirculating system with aerated water. Twenty percent of the water was exchanged daily with fresh water. The water temperature was maintained at $25 \pm 1{ }^{\circ} \mathrm{C}$. The photoperiod was $12 \mathrm{~h}$ light:12 $\mathrm{h}$ dark. The pale chubs were hand-fed to satiation once daily (at 8:00 am) for 4 weeks with tubifex. The feces and uneaten food were removed with a siphon at 9:00 am. The dissolved oxygen level was kept above $90 \%$ saturation. After 4 weeks of acclimation, experimental fish were tagged intraperitoneally with passive integrated transponder (PIT) tags under anesthesia by neutralized tricaine methane sulfonate (MS222, $50 \mathrm{mg} \mathrm{L}^{-1}$ ) and allowed to recover for 2 weeks. All individuals were used to measure personality. 
The present study was authorized by the Animal Care and Use Committee of the Key Laboratory of Animal Biology of Chongqing (permit number: Zhao-20161122-04).

\section{Experimental setup}

The experimental setup was similar to those described in Tang \& Fu (2019). Specifically, I used four rectangular tanks (length $\times$ width $\times$ height: $70 \times 35 \times 35 \mathrm{~cm}$ ) as arenas to measure spontaneous swimming behavior in the different contexts, as in previous studies (Liu \& Fu, 2017; Fig. 1). The arena was surrounded with an opaque canvas to eliminate visual stimuli from the observers during the experiments. The arena was separated into two subareas by an opaque plastic partition; the two subareas included an open area (length $\times$ width $\times$ height: $55 \times 35 \times 35 \mathrm{~cm}$ ) and a shelter area (length $\times$ width $\times$ height: $15 \times 35 \times 35 \mathrm{~cm}$ ), which provided a refuge with artificial plants. A small door (width $\times$ height: $10 \times 10 \mathrm{~cm}$ ) on the partition allowed fish to move freely from the shelter area to the open area in the boldness context, but it was closed in the exploration and activity (i.e., control) contexts. The water depth was maintained at $10 \mathrm{~cm}$ during the experiment. The behaviors of the test fish were recorded using a webcam (Logitech Pro 9000; Logitech Company, Suzhou, China) placed 1.5 m directly above the arena and connected to a remote monitor, and the experimental arena was illuminated by fluorescent lights.

\section{Experimental protocol}

The recordings were conducted in all four arenas at the same time from 8:00 to 17:00 $\mathrm{h}$. The fish were first recorded in the boldness context, then the exploration context, and finally the control context (see details below). The arenas were cleaned after the measurement of each individual.

For the boldness context, a fish was transferred into the shelter area and acclimated for 30 min; then, the door was gently opened by a remotely operated line, and the movement of the fish (only in the open area) was recorded for 30 min (at 15 frames per second). Pale chub individuals showed large variation in latency to enter the open area (ranging from nearly zero to $23 \mathrm{~min}$ ) and the percentage of time spent in the open area (18 to $97 \%$ ). Thus, the total duration of moving in the open area varied greatly among individuals. To record movement in the exploration context, fish remaining in the shelter area were gently chased into the open area of the arena and acclimated for 10 min with the small door 
127 between the open and hidden areas closed. Then, a novel object (a black, round, plastic ball with a 128 diameter of approximately $2 \mathrm{~cm}$, Liu \& Fu., 2017) was carefully placed on the arena bottom $40 \mathrm{~cm}$

129 from the small door (Fig. 1), and the movements of the fish were recorded by the webcam for 10 min.

130 For the control context, the novel object was removed, and the fish was again acclimated for 10 min

131 to eliminate the effect of the exploration context. Then, the movements of the fish were recorded for $13210 \mathrm{~min}$.

\section{Data calculation and analysis}

134 Spontaneous movements were recorded for all 80 individuals in the three contexts. However, the number of replicates differed among the tests because some files could not be read by the software due to technical problems.

137 The videos were imported into an automated tracking program (EthoVision XT 9, Nodus, Netherlands) after converting the $\mathrm{x}$ and $\mathrm{y}$ coordinates from pixels to $\mathrm{cm}$. The trajectories were smoothed using a weighted moving average with a window width of $0.5 \mathrm{~s}$ (Miller \& Gerlai, 2012). Then, the median speed while swimming (swimming speed above $1.75 \mathrm{~cm} \mathrm{~s}^{-1}$ ), percent time moving and median turning rate while swimming were calculated for all three contexts. I eliminated movements with speeds less than $1.75 \mathrm{~cm} \mathrm{~s}^{-1}$ to minimize potential system noise and small movements of the animal (body wobbles) according to previous studies (Tang et al., 2017; Tang \& Fu, 2019).

The swimming speed $\left(\mathrm{v}, \mathrm{cm} \mathrm{s}^{-1}\right)$ was calculated as follows:

$$
v(t)=\sqrt{(x(t)-x(t-1))^{2}+(y(t)-y(t-1))^{2}} / d
$$

where $x(t)$ and $x(t-1)$ and $y(t)$ and $y(t-1)$ are the $x$ and $y$ coordinates, respectively, of the measured fish at time $t$ and the time of the previous frame $(t-1)$ and $d$ is the length of the time interval (i.e., $0.5 \mathrm{~s}$, Miller \& Gerlai, 2012). The percent time moving was calculated as the percentage of time when the swimming speed was above $1.75 \mathrm{~cm} \mathrm{~s}^{-1}$. The turning rate $\left(\omega_{t}, \mathrm{rad} \mathrm{s}^{-1}\right)$ of an individual was calculated using the absolute centroid change (see details in Herbert-Read et al., 2013). This measure can be used to quantify exploratory as opposed to goal-directed behavior in fish and is calculated as follows (loannou, Singh \& Couzin, 2015; Sumpter et al., 2018):

$$
\omega_{t}=\frac{\cos ^{-1}\left(c_{t} \cdot c_{t+1}\right)}{\Delta t}
$$


154 In brief, $\theta_{t}$ is the orientation of the fish at time step $i$ measured relative to the positive $x$ axis of the 155 coordinate system. Let $\mathbf{c}_{t}=\cos \theta_{t}+\sin \theta_{t}$ be the unit vector pointing in the direction of $\theta$ at time step $156 i$. Then, the change in the orientation of the fish from time step $t$ to time step $t+1$ is given by

$157 \cos ^{-1}\left(\boldsymbol{c}_{t} \bullet \boldsymbol{c}_{t+1}\right)$. Again, I calculated the median turning speed of each individual only when the 158 swimming speed was above $1.75 \mathrm{~cm} \mathrm{~s}^{-1}$.

159 For the exploration trials, I also calculated two variables that are commonly used to test for 160 differences in exploratory tendency in fish, i.e., the mean distance to the object and the latency to 161 explore the novel object in the exploration context (Adriaenssens \& Johnsson, 2011; Mazué \& Godin, 162 2015). The distance $(\mathrm{cm})$ was calculated as

$D_{i}=\sqrt{\left(x_{i}-x_{n}\right)^{2}+\left(y_{i}-y_{n}\right)^{2}}$

where $x_{i}$ and $y_{i}$ denote the coordinates of the fish and $x_{n}$ and $y_{n}$ denote the coordinates of the object. The mean value of all frames was used for analysis. The latency to explore the novel object was defined as the amount of time it took for the fish to first swim within $7 \mathrm{~cm}$ (approximately 1 body length) of the object. To assess whether the fish were responding to the novel object, I also measured mean distance and latency in relation to a virtual object in the same location in the control context. The calculation assumed that the coordinates of both the novel and virtual objects were located at the center of the object. Thus, I assumed that the $1 \mathrm{~cm}$ radius of the novel or virtual object would have little effect on measures of distance and latency to explore.

One-sample Kolmogorov-Smirnov tests indicated that median swimming speed, median turning rate and distance to the novel object were normally distributed, whereas percent time moving and latency to explore the novel (or virtual) object were not normally distributed. I used Pearson correlation to compare normally distributed measures and Spearman's rank correlation to compare measures that were not normally distributed. In the case of significant correlations, the relationships were also examined using linear regressions. The effect of measurement context on median swimming speed and median turning rate was tested by a linear mixed model (LMM) using fish ID as a random factor. This was followed by a paired t-test to assess the difference in median swimming speed between any two contexts. The difference in percent time moving was assessed by a nonparametric Wilcoxon 
181

182

183

184

185

186

187

188

189

190

191

192

193

194

195

196

197

198

199

200

201

202

203

204

205

206

207

matched-pairs test. The distance from the real or virtual novel object was compared with a paired ttest, whereas the latency to explore the object was compared with a Wilcoxon matched-pairs test. The program SPSS 17 was used for data analysis. $P$-values $<0.05$ were considered statistically significant, and all the data are presented as the mean \pm S.E.

\section{RESULTS}

\section{Effect of measurement context on spontaneous movement traits}

The median swimming speed (Pearson correlation, $P<0.001$ ), percent time moving (Spearman correlation, $P<0.001$ ) and median turning rate (Pearson correlation, $P<0.001$ ) measured in the control context were positively correlated with those measured in the other two contexts (Table 1; Fig. 2).

Measurement context had a significant effect on median swimming speed $\left(\mathrm{LMM}, F_{2,150.29}=70.637\right.$, $P<0.001)$ but no effect on median turning rate $\left(L M M, F_{2,149.17}=0.263, P=0.796\right)$ (Fig. 3$)$. The median swimming speed of fish measured in the boldness context was significantly higher than that measured in both the exploration (paired t-test, $t_{73}=9.723, P<0.001$ ) and control (paired t-test, $t_{73}=10.398, P$ $<0.001)$ contexts. The percent time spent moving measured in the boldness context was significantly larger than that measured in the exploration context (Wilcoxon test, $z=-4.873, P<0.001$ ), whereas the latter was significantly larger than that measured in the control context (Wilcoxon test, $z=-5.083$, $P<0.001)$.

Difference in exploration variables between the exploration context and control context with a virtual object

The distance to the novel (or virtual) object (Pearson correlation, $\mathrm{R}=0.435, \mathrm{~N}=79, P<0.001$ ) and the latency to reach the novel (or virtual) object (Spearman correlation, $\mathrm{R}=0.329, \mathrm{~N}=78, P<0.001$ ) were positively correlated (Fig. 4).

The distance to the novel object in the exploration context showed no significant difference from that to the virtual object in the control context (paired t-test, $t_{78}=-0.838, P=0.405$ ) (Fig. 5A). However, it took a much shorter amount of time for the fish to first reach the novel object in the exploration context than for the fish to reach the virtual object in the control context (Wilcoxon test, $z=2.343, P<0.001$ ) 
208 (Fig. 5B).

209 DISCUSSION

210 The correlation of spontaneous swimming traits between the control and other two contexts

211 The values of activity variables, such as the median swimming speed, median turning rate and percent

212 time moving, measured in the control context were closely correlated with those measured in the 213 boldness and exploration contexts. This agrees with the results of a previous study showing that 214 variables such as median swimming speed and median turning rate were quite constant across 215 measurements or contexts in mosquitofish (Gambusia affinis) (Herbert-Read et al., 2013) and in 216 zebrafish (Danio rerio) (Toms \& Echevarria, 2014). These results suggest that researchers can 217 evaluate personality traits associated with activity using only boldness or exploration contexts and do 218 not need to perform activity measurements separately. It is worth noting that the correlations may be 219 related to factors other than individual differences because I measured the personality traits in only a 220 single test. For example, it is possible that similarities between traits are due to factors such as the 221 time of day, amount of time in the holding tank before testing, noise in the environment and stress of 222 capture from the holding tank. However, it is unlikely that the correlation was due to such factors, as a previous study found that the median swimming speed and time spent moving under spontaneous swimming were constant and showed high repeatability throughout the day (from 8:00 to 18:00 h) and that almost all personality traits (seven of eight traits) showed high repeatability between two tests on two consecutive days (Tang, 2019). Nevertheless, more investigations (e.g., multiple measurements in different contexts but with different orders) need to be conducted before drawing a solid conclusion about the feasibility of using activity in boldness or exploration contexts as an adequate substitute for a separate activity test.

\section{Comparison of spontaneous swimming traits across contexts}

231 Fish in the boldness context showed a 33\% higher median swimming speed and 35\% higher percent

232 time moving than those in the control context. The faster speeds and more time spent moving of pale 233 chubs in the boldness context could be because the fish could rely on returning to the shelter when 234 threatened, whereas they reduced activity (speed and time spent moving) in the activity context 
235

236

237

238

239

240

241

242

243

244

245

246

247

248

249

250

251

252

253

254

255

256

257

258

259

260

261

because a refuge was not available. For example, a previous study in juvenile seabass (Dicentrarchus labrax) showed that activity is reduced when perceived threat increases (Herbert-Read et al., 2017). However, the difference in swimming activity between boldness and control contexts might also be because some higher movement rates result from fish fleeing quickly back to the shelter after entering the arena. Finally, the differences between the boldness and control contexts might also be a result of test order or time in the arena because the three contexts were measured in the same order for all fish. For example, although the pale chubs were acclimated for 30 min (the acclimation period was long enough according to our previous observation) before measurement in the boldness context, one might argue that the fish were more stressed from being captured and hence moved and explored their environment more in the boldness context but then either tired or calmed down in the exploration and control contexts. This might be an alternative explanation for the reduction in swimming speed or percent time moving from the boldness to exploration or activity context. Future studies should consider the effect of order in the arena on personality measurements.

\section{The relationships of exploration variables between exploration and control contexts}

All variables measured in the exploration context were almost the same as those in the control context.

This suggests that the presence of so-called novel objects might not have been a stimulus for exploration in pale chubs in the present study. This was not due to the size and shape of the arena $(70 \times 35 \times 35 \mathrm{~cm})$ or novel object $(1 \mathrm{~cm}$ radius $)$ in the present study, as they were similar to those in previous studies. For example, a previous study in blue gourami (Trichogaster trichopterus) used a 40 $\times 60 \times 40 \mathrm{~cm}$ arena (Bisazza, Lippolis \& Vallortigara, 2001), and a $90 \times 30 \times 45 \mathrm{~cm}$ arena and 1.3cm-diameter novel object were used for the guppy (Poecilia reticulata) (De Serrana, Fong \& Rodd, 2016). A $50 \times 20 \times 36 \mathrm{~cm}$ aquarium and 1.8-cm-diameter novel object were used for zebrafish (LuconXiccato \& Dadda, 2014), and identical conditions were adopted in crucian carp (Liu \& Fu, 2017). Thus, the fact that previous studies as well as the present study found a positive relationship between activity variables such as speed (or percent time moving) and exploration variables (distance and latency to explore the novel object) might have occurred because more active fish spend more time swimming at higher speeds and hence show a shorter distance to the object and exploration latency than do less 
262 active individuals (Liu \& Fu, 2017; Tang \& Fu, 2019). This is reinforced by the results indicating that

263 the fish showed almost identical values in terms of the mean distance to novel objects in the same

264 location when measured in the control context (i.e., a virtual object). However, this might have occurred

265 because the pale chubs in the present study did not need to change their swimming behavior or

266 position in the arena to explore. Furthermore, the handling stress during experimental measurement

267 might also have resulted in a lack of change in swimming pattern in the fish when subjected to different

268 test contexts. The situation could have been reinforced, as pale chubs were measured individually in

269 the present study, whereas this fish species often forms shoals in the field. Recent studies in cyprinid

fish species such as qingbo (Spinibarbus sinensis) found that the responses of spontaneous activity patterns to threat varied profoundly when tested individually compared to those tested in groups (Wang Fu \& Fu, 2019; Xu, Fu \& Fu, 2019). Interestingly, the time it took for the fish to first reach the object in the exploration context was much shorter than that in the control context; i.e., the fish encountered the object earlier in the exploration context. This suggests that pale chubs not only showed no neophobic tendency but also exhibited exploratory behavior towards the 'novel' object and that latency can be used as an exploration indicator in the pale chub under the conditions used in the present study (Galhardo, Vitorino \& Oliveira, 2012). This might be because the pale chub perceived the 'novel' object as something that was potentially rewarding and worthy of exploration, such as food. The lack of a difference in the spontaneous activity pattern of the pale chub between the exploration and control contexts might have occurred because the novel object was too simple and the pale chub performed only superficial inspection or contact with the object and then exhibited a spontaneous swimming pattern similar to that observed in the control context. Nevertheless, this study suggests that fish behaviorists should be cautious regarding explanations pertaining to exploration (or neophobia) indicators previously used in other personality studies, such as the distance to an object (Wang et al., 2019; Wang, 2019). Researchers at least need to test the reliability of such measurements, for example, by comparing spontaneous movement trajectories in fish between exploration and control contexts, as in the present study. The testing of other variables under different experimental setups, such as the number of times that individual 
guppies traveled between containers connected by pipes (Brown \& Irving, 2014), might be more appropriate for the measurement of exploration tendency.

\section{1}

292

\section{CONCLUSIONS}

In conclusion, the pale chubs showed strong positive correlations in median swimming speed, median turning rate and percent time moving between the control and other two contexts, which suggests that fish behaviorists can use such traits from either boldness or exploration measurement tests rather than having to conduct additional tests. Further analysis revealed that the latency to explore an object might be a reliable indicator of exploration, whereas a traditionally used variable such as distance to a novel object might not be an appropriate indicator, at least for the pale chub under the conditions in the present study.

Acknowledgments This work was supported by the National Natural Science Foundation of China (No. 31670418). We would like to thank Dr. Donald Kramer, Dr. Christos loannou and one anonymous reviewer for insightful comments and constructive suggestions for statistical analysis and writing that greatly improved the manuscript.

\section{References}

Adriaenssens B, Johnsson Jl. 2011. Shy trout grow faster: exploring links between personality and fitness-related traits in the wild. Behavioral Ecology 24:135-143

Bevan PA, Gosetto I, Jenkins ER, Barnes I, Ioannou CC. 2018. Regulation between personality traits: individual social tendencies modulate whether boldness and leadership are correlated. Proceedings of the Royal Society B 285:20180829

Bell AM, Hankison SJ, Laskowski KL. 2009. The repeatability of behaviour: a meta-analysis. Animal Behaviour 77:771-783

Bisazza A , Lippolis G, Vallortigara G. 2001. Lateralization of ventral fins use during object exploration in the blue gourami (Trichogaster trichopterus). Physiology and Behavior 72(:575-578.

Brown C, Irving E. 2014. Individual personality traits influence group exploration in a feral guppy population. Behavioral Ecology 25:95-101

Burns JG. 2008. The validity of three tests of temperament in guppies (Poecilia reticulata). Journal of Comparative Psychology122:344-356

Couzin ID, loannou CC, Demirel G, Gross T, Torney CJ, Hartnett A, Conradt L, Levin SA, Leonard NE. 2011. Uninformed individuals promote democratic consensus in animal groups. Science 334:1578-1580

De Serrano AR, Fong C, Rodd FH. 2016. Effects of methylphenidate on responses to novelty in a teleost fish (Poecilia reticulata). Behavioural Brain Research 302:53-59

Galhardo L, Vitorino A, Oliveira RF. 2012. Social familiarity modulates personality trait in a cichlid fish. Biology Letters 8:936-938

Heg D, Schuürch R, Roghenberger S. 2011. Behavioral type and growth rate in a cichlid fish. 
Behavioral Ecology 22:1227-1233

Herbert-Read JE, Krause S, Morrell LJ, Schaerf TM, Krause J, Ward AJW. 2013. The role of individuality in collective group movement. Proceedings of the Royal Society B 280:20122564

Herbert-Read JE, Kremer L, Bruintjes R, Radford AN, loannou CC. 2017. Anthropogenic noise pollution from pile-driving disrupts the structure and dynamics of fish shoals. Proceedings of the Royal Society B $284: 20171627$

loannou CC, Singh M, Couzin ID. 2015. Potential leaders trade off goal-oriented and socially oriented behavior in mobile animal groups. American Naturalist 186:284-293

Jolles JW, Boogert NJ, Sridhar VH, Couzin ID, Manica A. 2017. Consistent individual differences drive collective behavior and group functioning of schooling fish. Current Biology 27:2862-2868

Jolles JW, Briggs H, Araya Y, Boogert NJ. 2019. Personality, plasticity and predictability in sticklebacks: bold fish are less plastic and more predictable than shy fish. Animal Behaviour 154:193-202

Liu S, Fu SJ. 2017. Effects of food availability on metabolism, behaviour, growth and their relationships in a triploid carp. Journal of Experimental Biology 220:4711-4719

Lucon-Xiccato T, Dadda M. 2014. Assessing memory in zebrafish using the one-trial test. Behavioural Processes 106:1-4

Martins EP, Bhat A. 2014. Population-level personalities in zebrafish: aggression-boldness across but not within populations. Behavioral Ecology 25:368-373

Mazué GPF, Godin JGJ. 2015. Boldness-exploration behavioural syndrome: interfamily variability and repeatability of personality traits in the young of the convict cichlid (Amatitlania siquia). Behavioral Ecology 26:900-908

Miller N, Gerlai R. 2012. Automated tracking of zebrafish shoals and the analysis of shoaling behavior. In: Kalueff AV, Stewart AM, eds. Zebrafish protocols for neurobehavioral research. New York, Humana Press, pp 217-230

Réale D, Garant D, Humphries MM, Bergeron P, Careau V, Montiglio PO. 2010. Personality and the emergence of the pace-of-life syndrome concept at the population level. Proceeding of the Royal Society B 365:4051-4063

Sih A, Cote J, Evans M, Fogarty S, Pruitt J. 2012. Ecological implications of behavioral syndromes. Ecology Letters 15:278-289

Sih A, Bell A, Johnson JC. 2004. Behavioral syndromes: an ecological and evolutionary overview. Trends in Ecology and Evolution 19:372-378

Smith BR, Blumstein DT. 2008. Fitness consequences of personality: a meta-analysis. Behavioral Ecology 19:448-455

Sumpter DJT, Szorkovszky A, Kotrschal A, Kolm N, Herbert-Read JE. 2018. Using activity and sociability to characterize collective motion. Philosophical Transactions of Royal Society B 373:20170015

Tang J. 2019. The personality of pale chub (Zacco platypus). Master Degree thesis. Chongqing Normal University Chongqing. China

Tang ZH, Fu SJ. 2019. Qingbo (Spinibarbus sinensis) personalities and their effect on shoaling behavior. Acta Ethologica 22:135-144

Tang ZH, Wu H, Huang Q, Kuang L, Fu SJ. 2017. The shoaling behavior of two cyprinid species in conspecific and heterospecific groups. PeerJ 5:e3397

Tang ZH, Wu QY, Fu SJ. 2018. Inspection behaviour and inter-individual cooperation in juvenile qingbo: the effects of prior predator exposure and food deprivation. Journal of Ethology 36:181190

Toms CN, Echevarria DJ. 2014. Back to basics: searching for a comprehensive framework for exploring individual differences in zebrafish (Danio rerio) behavior. Zebrafish 11: 325-340

Wang L. 2019. The effect of food abundance on standard metabolic rate, personality and shoal behavior of pale chub (Zacco platypus). Master Degree thesis. Chongqing Normal University Chongqing. China

Wang L, Tang JY, Qin YL, Zeng LQ, Peng JL, Fu SJ. 2019. Effect of starvation on energy metabolism, fish behavior, and schooling behavior of Spinibarbus sinensis. Acta Ecologica Sinica 39:1095-

Peer) reviewing PDF | (2019:09:41698:2:0:NEW 30 Jan 2020) 
$381 \quad 1104$

382 Wang Y, Fu SJ, Fu C. 2019. Behavioral adjustments to prior predation experience and food deprivation 383 of a common cyprinid fish species vary between singletons and a group. PeerJ 7: e7236

$\mathrm{Xu} \mathrm{JJ}, \mathrm{Fu}$ SJ, Fu C. 2019. Physiological and behavioral stress responses to predators are altered by prior predator experience in juvenile qingbo (Spinibarbus sinensis). Biology Open 8:bio041012 


\section{Table $\mathbf{1}$ (on next page)}

The statisticalresults of correlation between the control and other two contexts

Pearson or Spearman (percent time moving) correlations between measurements conducted under the control context and those conducted under either boldness or exploration contexts. 
1 Table 1 Correlations between measurements of activity in pale chub conducted in the control context 2 and those conducted in either boldness or exploration contexts. Correlations coefficients were 3 calculated using Pearson $(R)$ or Spearman's $\left(R_{s}\right)$ correlation coefficients.

4

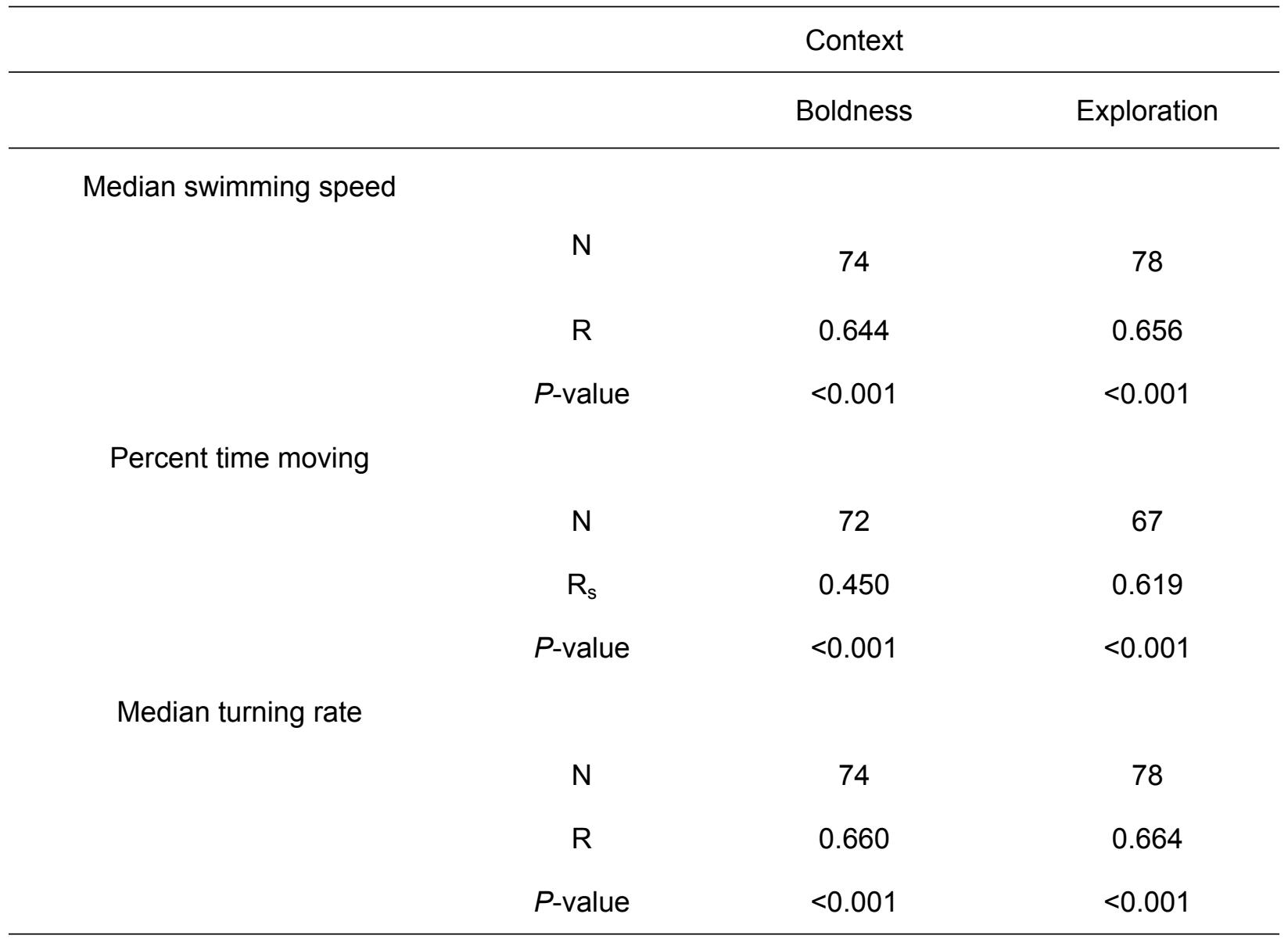


Figure 1

Design of the experimental aquarium used for the sponteneous swimming activyt in boldness, exploration and activity contexts in the study.

A) shelter (length $\times$ width $\times$ height: $15 \times 35 \times 35 \mathrm{~cm}$ ); B) open area (length $\times$ width $\times$ height: $55 \times 35 \times 35 \mathrm{~cm})$; C) removable opaque PVC divider; and D) small door $(10 \times 10 \mathrm{~cm})$

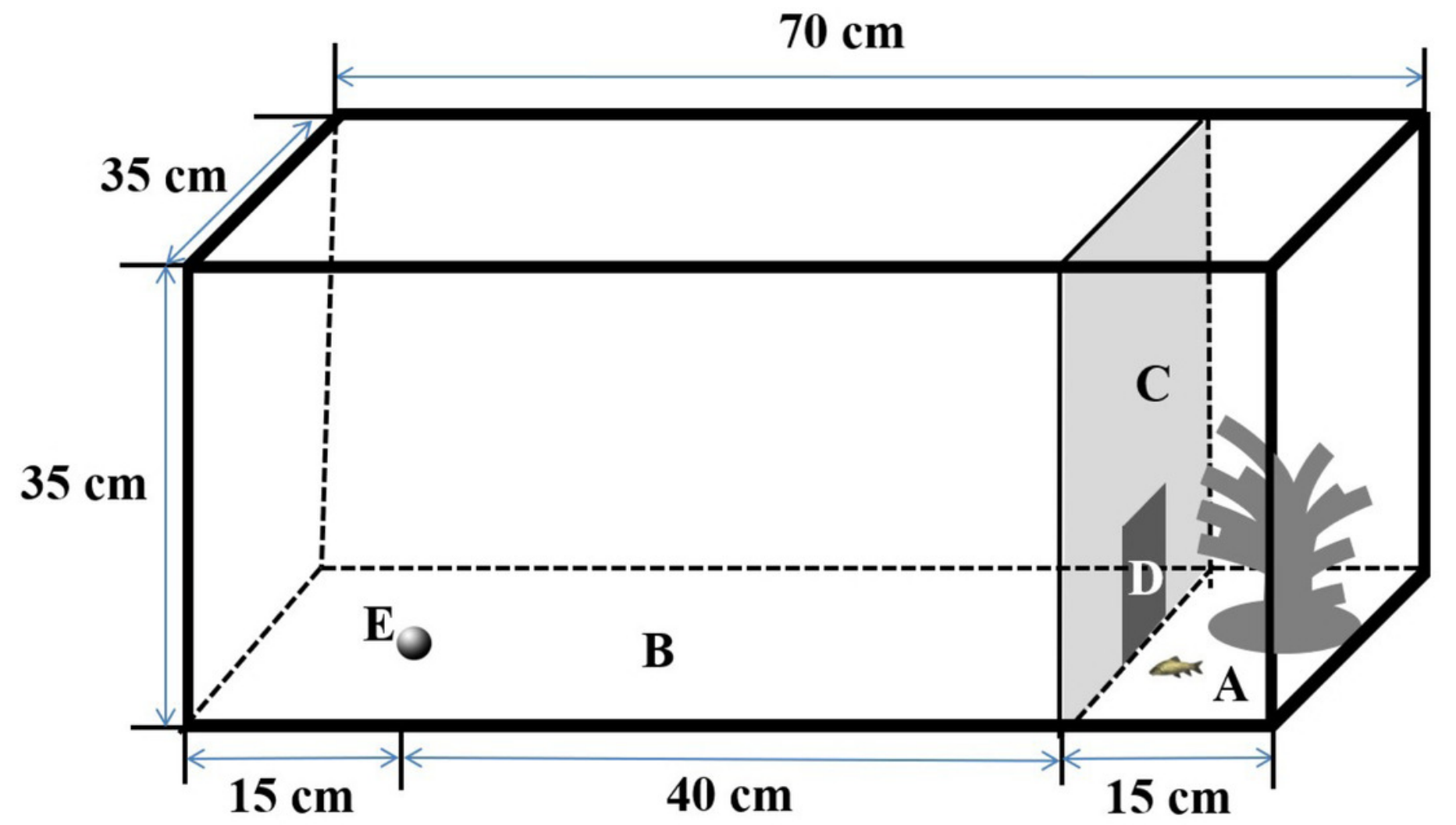


Figure 2

Correlations between the control context and two other contexts, boldness (red dots) and exploration (blue dots) for three measures of activity

[median swimming speed (A), percent time moving (B) and median turning rate $(C)$ ] in pale chubs. Dotted lines represent the relationships between two different contexts using linear regressions. 


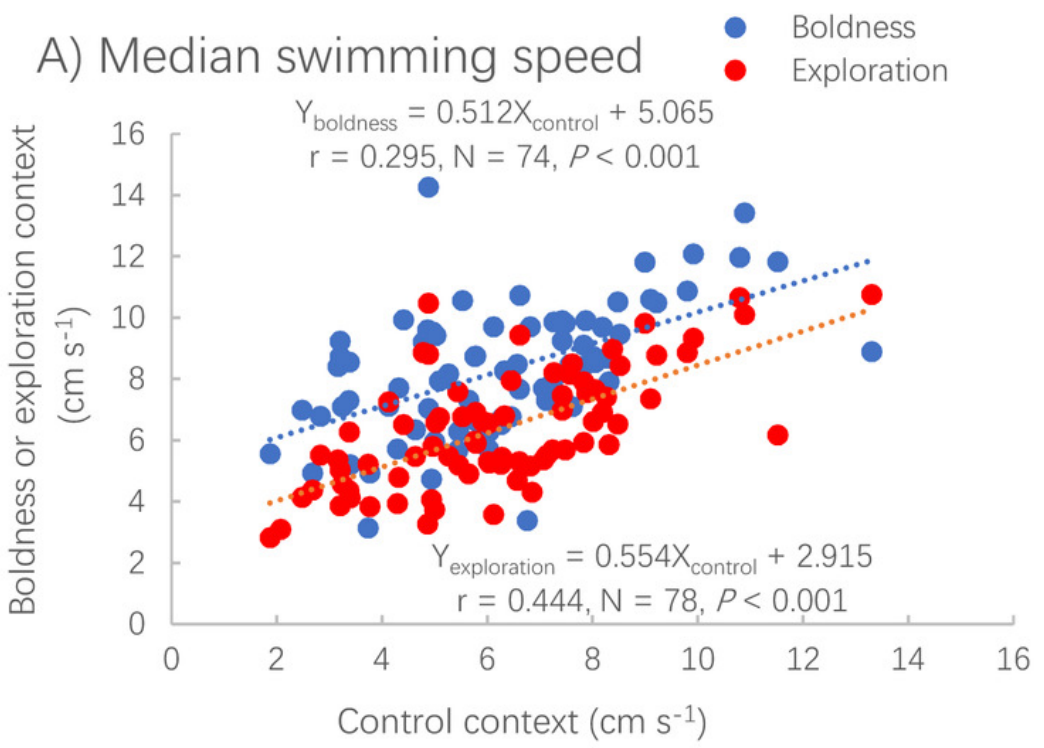

B) Percent time moving $\quad \bullet$ Boldness
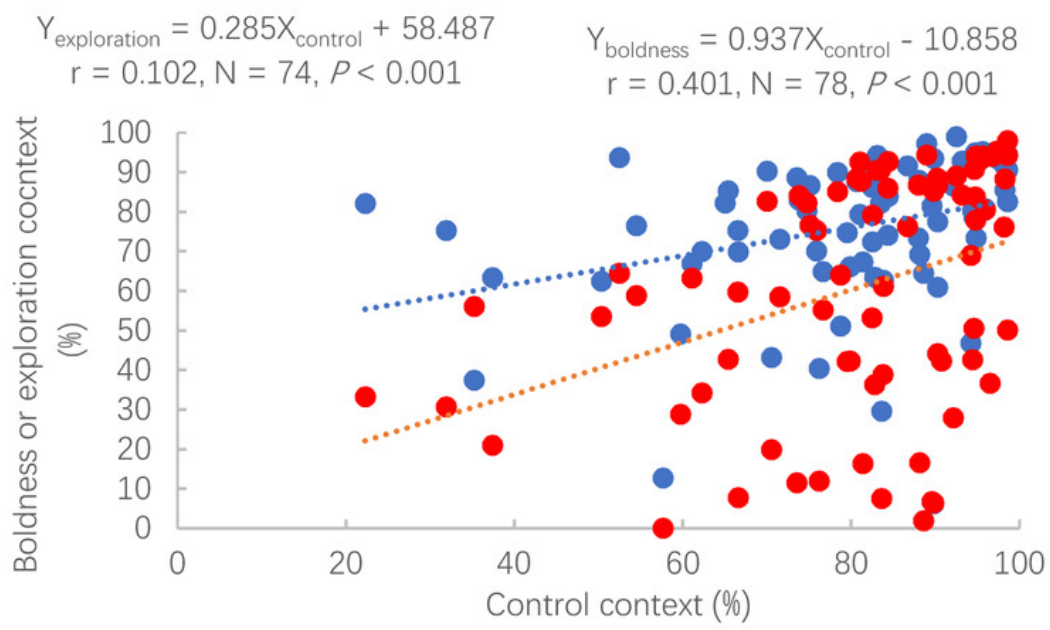
C) Median turning rate
- Boldness
- Exploration

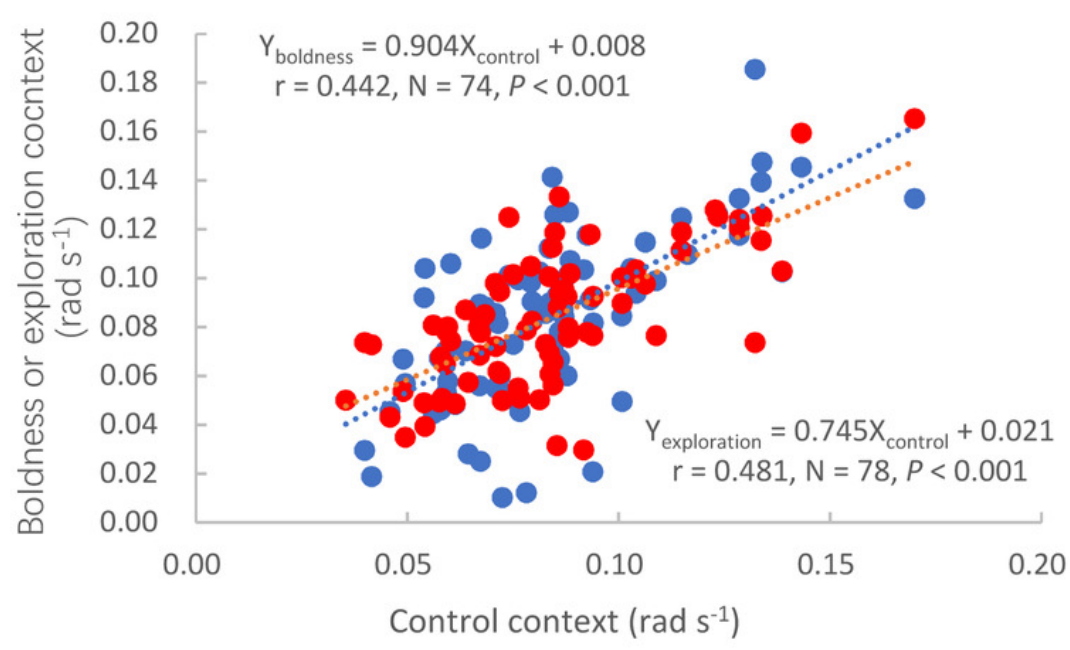




\section{Figure 3}

Mean \pm S.E. of four measures of spontaneous swimming activity

[median swimming speed (A), percent time moving $(B)$ and median turning rate $(C)$ ] in pale chubs in the boldness $(N=74)$, exploration $(N=78)$ and control $(N=79)$, contexts. Bars with different letters are significantly different $(P<0.05)$. 
A) Median swimming speed

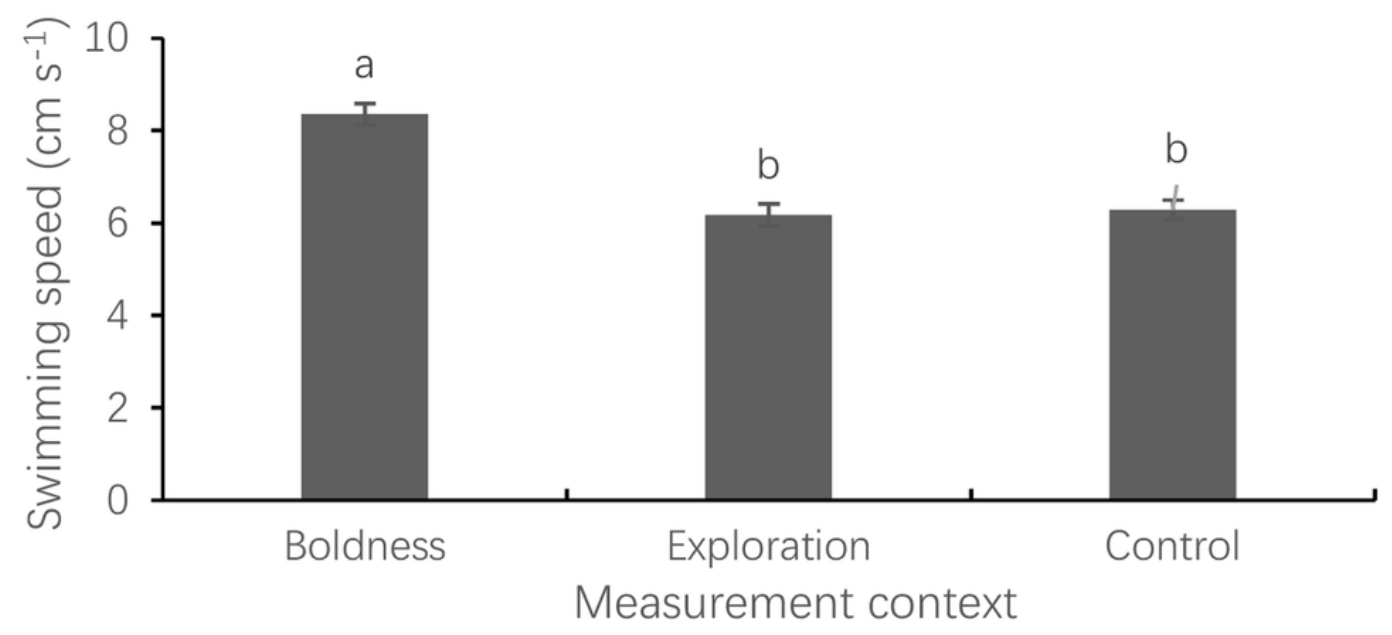

B) Percent time moving

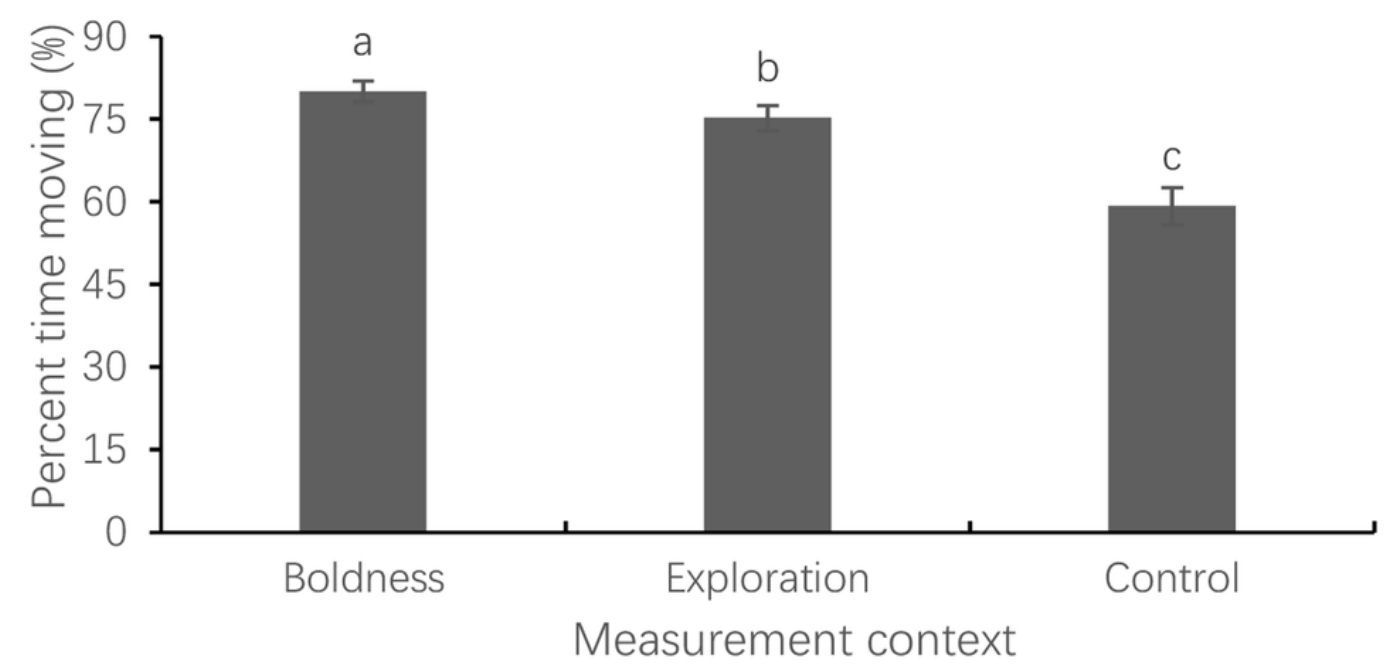

C) Median turning rate

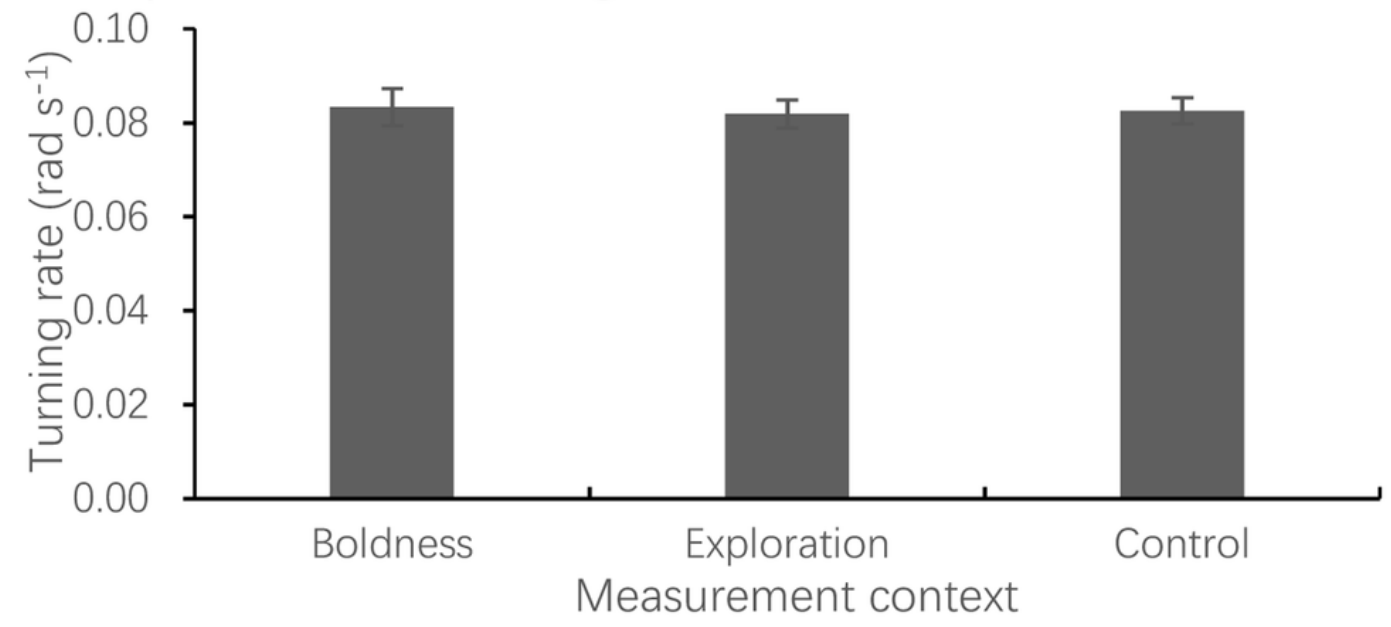


Figure 4

Correlations between the exploration and control contexts

Correlations between the exploration and control contexts for measures of distance to the novel object (A) and latency to the object (B). Dotted lines represent the relationships between two different contexts using linear regressions. 
A) Mean distance to object

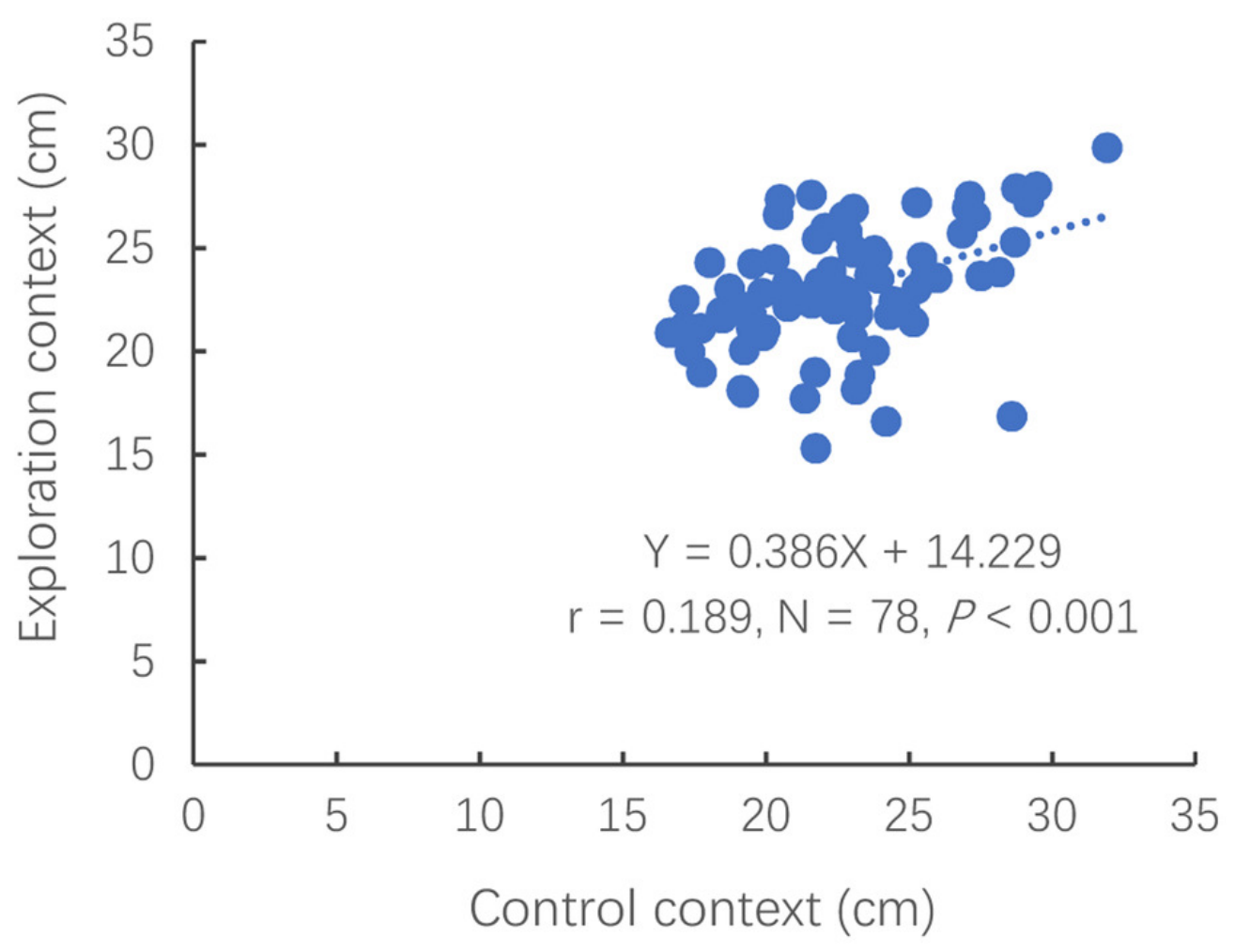

B) Latency to exploration

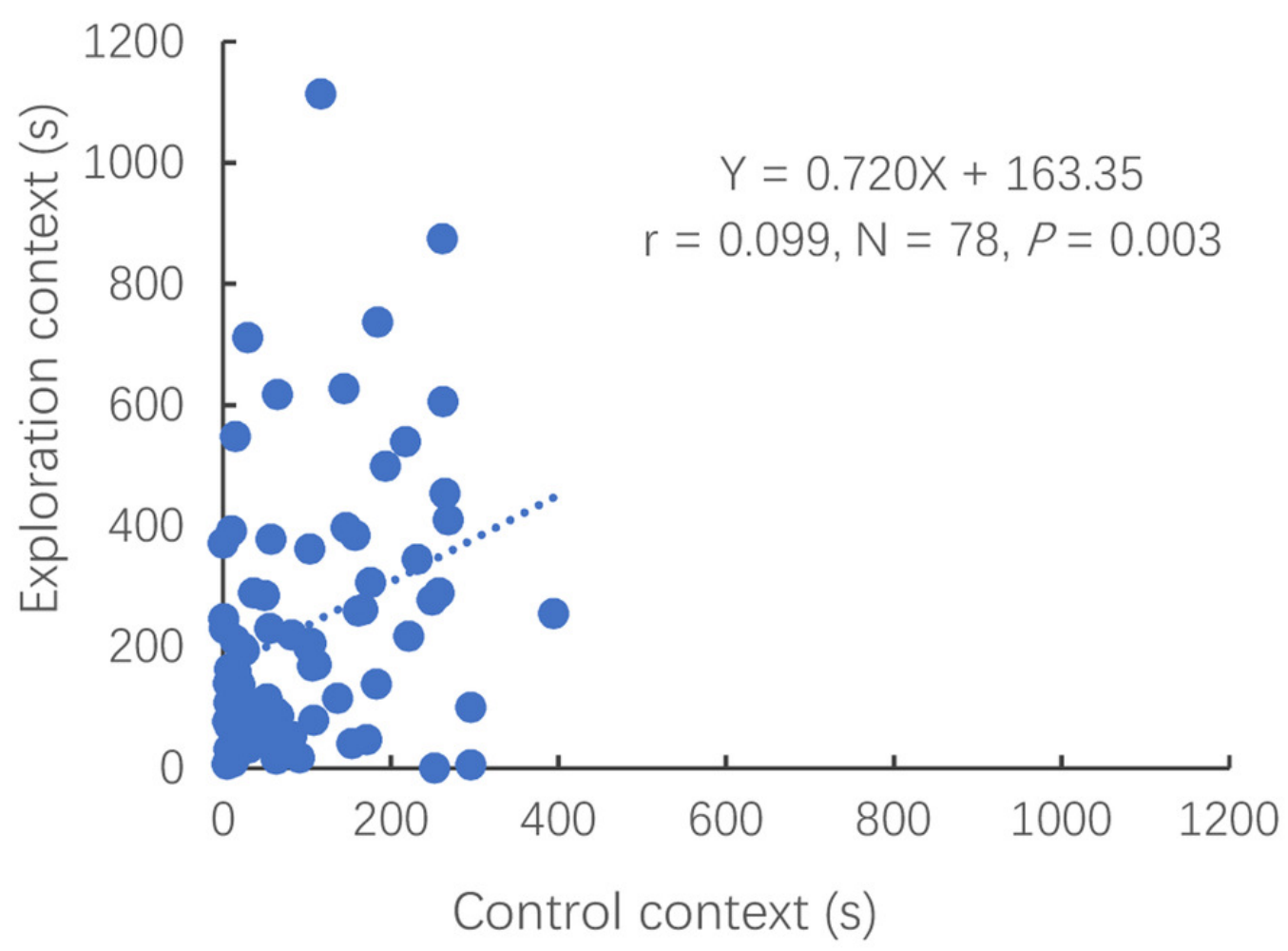


Figure 5

Mean distance (A) and latency to exploration (B) under the exploration context and the virtual object under the activity context

(mean \pm S.E., $N=78$ ). Bars with different letters are significantly different $(P<0.05)$. 

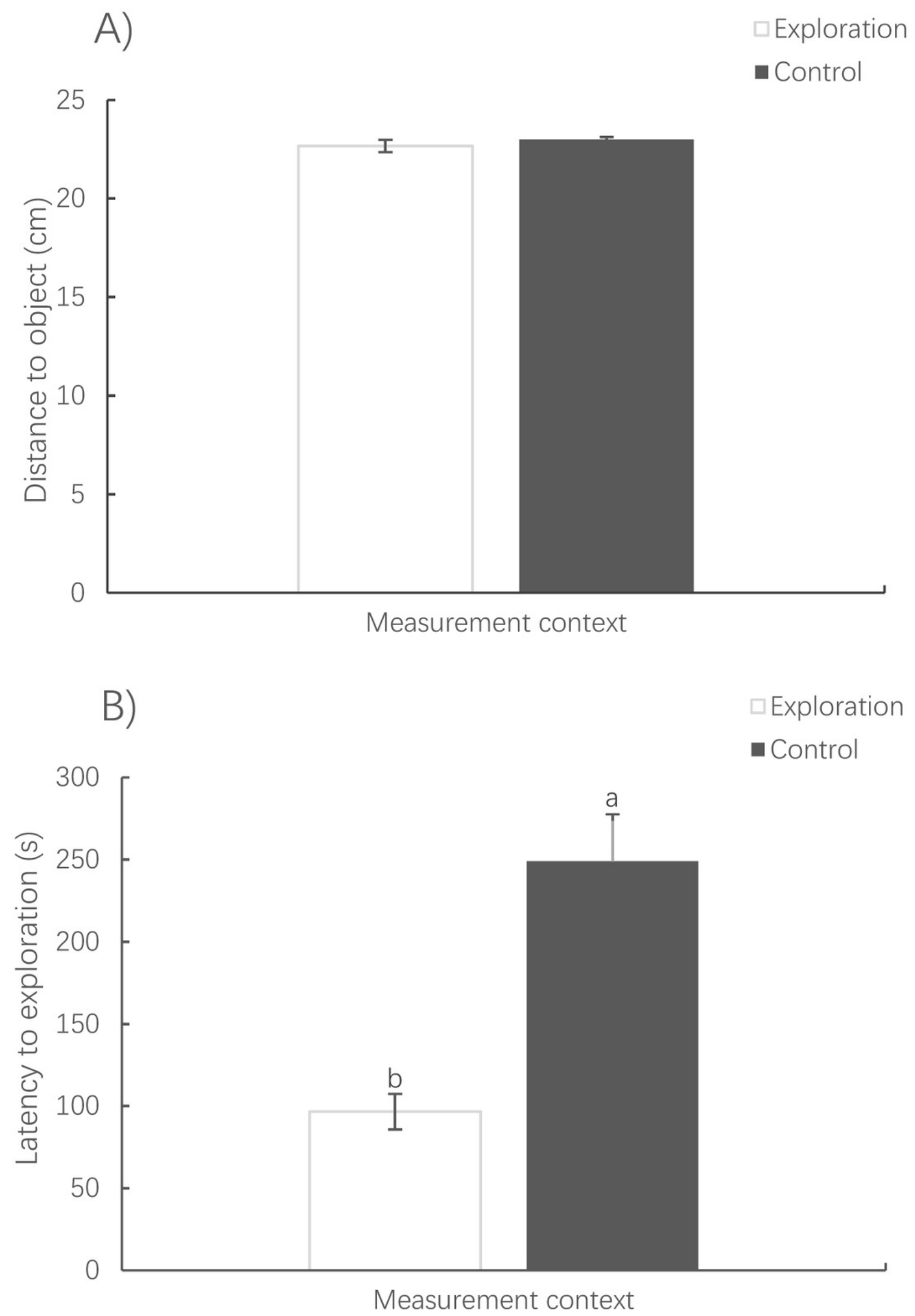